\title{
The time is right for an international PCD disease registry: insight and ongoing research activities
}

\author{
To the Editor:
}

We read the editorial entitled "The time is right for an international primary ciliary dyskinesia disease registry" with interest [1]. In it, HAVER [1] discussed our recent article on the International Primary Ciliary Dyskinesia Cohort (iPCD Cohort) [2] and stressed three aims important to primary ciliary dyskinesia (PCD) research: the development of a prospective international PCD registry, the standardisation of data collection and the achievement of an international diagnostic consensus. We support these aims and here highlight ongoing activities that support them.

Patient registries collecting relevant epidemiological data are key instruments for research on rare diseases that improve patient care and healthcare planning [3]. To answer the need for collaborative research in PCD, the European Union's Seventh Framework Programme project BESTCILIA and the BEAT-PCD COST Action have taken several initiatives.

BESTCILIA allowed the setup of both the retrospective iPCD Cohort [2] and a prospective international PCD registry [4]. The iPCD Cohort [2] is the first effort to combine all available datasets on PCD patients collected by published research studies, local case series and national registries. It thus includes patients diagnosed since the 1960s. Due to the retrospective nature of the iPCD Cohort and the evolution of diagnostics since the 1960s, it was not possible, as HAVER [1] noted, to achieve complete consensus on the diagnostic data and clinical information for all patients. However, extensive discussions among all researchers produced agreement on the most relevant variables.

The international PCD registry [4] was developed in collaboration between European and North American researchers and PCD support groups. It collects prospective PCD patient data and unites existing national PCD registries. The registry items, developed as a consensus result of the BESTCILIA consortium, collect diagnostic information in a standardised format. Despite this, the registry does not contain information on all available diagnostic tests (nasal nitric oxide, high-speed video microscopy, transmission electron microscopy, genetics, immunofluorescence and others) for each patient because not every contributing centre can use every diagnostic method. In addition, the number of tests needed to make a diagnosis varies depending on patient phenotype.

Clinical information reported in medical research is indeed still far from standardised, and clinical assessment and descriptions of symptoms of PCD patients vary widely [5]. For this reason, a multidisciplinary group of experts working in the framework of the COST Action BEAT-PCD [6] is further standardising data collection with two ongoing projects: the development of disease-specific standardised proformas for assessment of clinical data in PCD routine care and a prospective observational study (PROVALF) evaluating intraindividual variation of lung function parameters in stable PCD patients. Both projects aim to improve availability of standardised longitudinal data of PCD patients for use in clinical care and research.

Given the need for diagnostic consensus as a base for a PCD registry, this is a crucial step. However, the process is complicated by the heterogeneous nature of PCD and limited evidence on the validity of existing diagnostic techniques. The recent European Respiratory Society (ERS) Task Force guidelines for the diagnosis of PCD [7] summarised the level of consensus it was possible to reach regarding the use of

@ERSpublications

It is important to use all available data for PCD research while we strengthen efforts to reach diagnostic consensus http://ow.ly/N7SP30btbVY

Cite this article as: Goutaki M, Halbeisen FS, Kuehni CE. The time is right for an international PCD disease registry: insight and ongoing research activities. Eur Respir J 2017; 49: 1700357 [https://doi.org/ 10.1183/13993003.00357-2017]. 
diagnostic tests to confirm or exclude PCD. Although this is an important step, we still deal with diagnostic challenges such as variation in the availability of tests between countries and the lack of standardisation of some tests. This is the reality even in countries with well-developed health services. In the USA, high-speed video microscopy is rarely used, while in Europe, the clinical availability of genetic testing is limited.

In the iPCD Cohort, we applied the definitions set by the ERS Task Force to classify patients. Clinical features continue to play an important role in the diagnosis of PCD given existing tests [7]. The BESTCILIA consortium thus decided to include all patients followed up as PCD by the participating centres and then stratify data analyses according to diagnostic certainty. To further improve diagnostic information in the iPCD Cohort, we encourage participating centres to repeat diagnostic testing in older patients using state-of-the-art techniques and to update diagnostic data in the iPCD datasets.

Achieving diagnostic consensus and collecting prospective data from many patients are jointly necessary but both can take time. We therefore stress the importance of using all available data for research in rare diseases such as PCD so as not to let the perfection of datasets retard research advances that can help patients. Information already included in datasets such as the iPCD Cohort can offer valuable insight into the disease and inform the development of prospective studies to come.

Myrofora Goutaki $^{1,2}$, Florian S. Halbeisen ${ }^{1}$ and Claudia E. Kuehni ${ }^{1}$

${ }^{1}$ Institute of Social and Preventive Medicine, University of Bern, Bern, Switzerland. ${ }^{2}$ Paediatric Respiratory Medicine, Children's University Hospital of Bern, University of Bern, Bern, Switzerland.

Correspondence: Myrofora Goutaki, Paediatric Respiratory Medicine, Children's University Hospital of Bern, University of Bern, Finkenhubelweg 11, 3012 Bern, Switzerland. E-mail: myrofora.goutaki@ispm.unibe.ch

Received: Feb 202017 | Accepted: March 052017

Support statement: The development of the iPCD Cohort has been funded from the European Union's Seventh Framework Programme under EG-GA No.35404 BESTCILIA: Better Experimental Screening and Treatment for Primary Ciliary Dyskinesia. Primary Ciliary Dyskinesia research at the Institute of Social and Preventive Medicine, Bern, is supported by a Swiss National Science Foundation grant (320030_173044) and national funding from the Lung Leagues of Bern, St Gallen, Vaud, Ticino and Valais, and the Milena-Pro Kartagener Foundation. The researchers participate in the network COST Action BEAT-PCD: Better Evidence to Advance Therapeutic options for PCD (BM 1407). Funding information for this article has been deposited with the Crossref Funder Registry.

Conflict of interest: None declared.

Acknowledgements: We want to thank all the patients in the iPCD Cohort and their families, and we are grateful to the PCD patient organisations who collaborated closely with us. We thank all the researchers who collaborate with us in the iPCD Cohort and other BEAT-PCD projects. We also thank Christopher Owen Ritter from the Institute of Social and Preventive Medicine at the University of Bern, Bern, Switzerland, for his editorial suggestions.

\section{References}

1 Haver K. The time is right for an international primary ciliary dyskinesia disease registry. Eur Respir J 2017; 49: 1602143.

2 Goutaki M, Maurer E, Halbeisen FS, et al. The international primary ciliary dyskinesia cohort (iPCD Cohort): methods and first results. Eur Respir J 2017; 49: 1601181.

3 Orphanet. Rare Disease Registries in Europe. www.orpha.net/orphacom/cahiers/docs/GB/Registries.pdf Date last updated: January 2016.

4 Werner C, Lablans M, Ataian M, et al. An international registry for primary ciliary dyskinesia. Eur Respir J 2016; 47: 849-859.

5 Goutaki M, Meier AB, Halbeisen FS, et al. Clinical manifestations in primary ciliary dyskinesia: systematic review and meta-analysis. Eur Respir J 2016; 48: 1081-1095.

6 Rubbo B, Behan L, Dehlink E, et al. Proceedings of the COST action BM1407 inaugural conference BEAT-PCD: translational research in primary ciliary dyskinesia - bench, bedside, and population perspectives. BMC Proceed 2016; 10: Suppl. 9, 66 .

7 Lucas JS, Barbato A, Collins SA, et al. European Respiratory Society guidelines for the diagnosis of primary ciliary dyskinesia. Eur Respir J 2017; 49: 1601090. 